\title{
Effect of Dual Coplanar Electrodes on Mercury-Free Flat Fluorescent Lamps for Liquid Crystal Display
}

\author{
Hyoung-Bin Park, Seong-Eui Lee, Gi Young Kim, Young Dong Lee, and Kyung Cheol Choi, Member, IEEE
}

\begin{abstract}
The effects of dual coplanar electrodes on a Hg-free flat fluorescent lamp were studied. For a dual coplanar lamp, brightness and efficacy were improved by $80 \%$ compared to a conventional coplanar lamp. The improvement is regarded as the result of reduced diffusion loss and the effective usage of the discharge volume in the dual coplanar lamps. An efficacy of $35.9 \mathrm{~lm} / \mathrm{w}$ $\left(14900 \mathrm{~cd} / \mathrm{m}^{2}\right)$ was achieved for 250 torr of a $\mathrm{Xe}(30 \%) / \mathrm{Ne}(70 \%)$ gas mixture, by a $20-\mathrm{kHz}$ ac pulse driving.
\end{abstract}

Index Terms-Dual coplanar electrodes, mercury-free flat fluorescent lamp.

\section{INTRODUCTION}

$\mathbf{R}$ ECENTLY, Hg-free flat fluorescent lamps have been widely studied as potential pollution-free high-luminance long-lifetime liquid crystal display (LCD) back-lights [1]-[4]. It has been common to use a pulsed operation to improve the ultraviolet (UV) generation efficiency and/or to prevent discharge contraction. Xenon is widely used, sometimes together with buffer gases, due to its low reactivity and high efficiency. Ikeda et al. [1] developed small-sized coplanar flat discharge lamps for use in such systems as car navigation, where a discharge was developed between a pair of coplanar electrodes by ac pulse driving. They achieved a system efficacy of $21 \mathrm{~lm} / \mathrm{W}$ at $10000 \mathrm{~cd} / \mathrm{m}^{2}$ for a $5.2^{\prime \prime} \mathrm{Xe}$ discharge lamp. For larger-sized back-lights, Ilmer et al. [2] developed a lamp composed of hundreds of uniformly distributed micro-discharges to prevent discharge contraction. The highest system efficacy in their case was around $28 \mathrm{~lm} / \mathrm{W}$, at $7500 \mathrm{~cd} / \mathrm{m}^{2}$. In their investigation, triangular discharge patterns were made between tipped cathodes and paired anodes by a dc pulse operation. We developed a lamp which comprises a pair of plane parallel electrodes, one on the front and the other on the bottom glass plate [5]. An 18" $\mathrm{Hg}$-free flat fluorescent lamp was driven by an ac sine wave.

In this study, we introduce the dual coplanar electrode flat fluorescent lamp, which comprises two pairs of electrodes, one pair on the outside surface of the front, and one on the bottom glass plate. In essence, this is the sum of two coplanar electrode lamps, one having the electrodes on the front plate and the other having them on the bottom plate. It demands less voltage to turn on, and is more stable than a conventional coplanar lamp. In

Manuscript received August 31, 2005; revised November 5, 2005.

H.-B. Park, G. Y. Kim, and Y. D. Lee are with the Devices Laboratory, Samsung Advanced Institute of Technology, Suwon 440-600, Korea.

S.-E. Lee is with the Department of Advanced Materials Engineering, Korea Polytechnic, Kyonggi Do 429-793, Korea.

K. C. Choi is with the Department of Electrical Engineering \& Computer Science, Korea Advanced Institiue of Science and Technology (KAIST), Daejeon 305-701, Korea (e-mail: kyungcc@ee.kaist.ac.kr).

Digital Object Identifier 10.1109/JDT.2005.862018

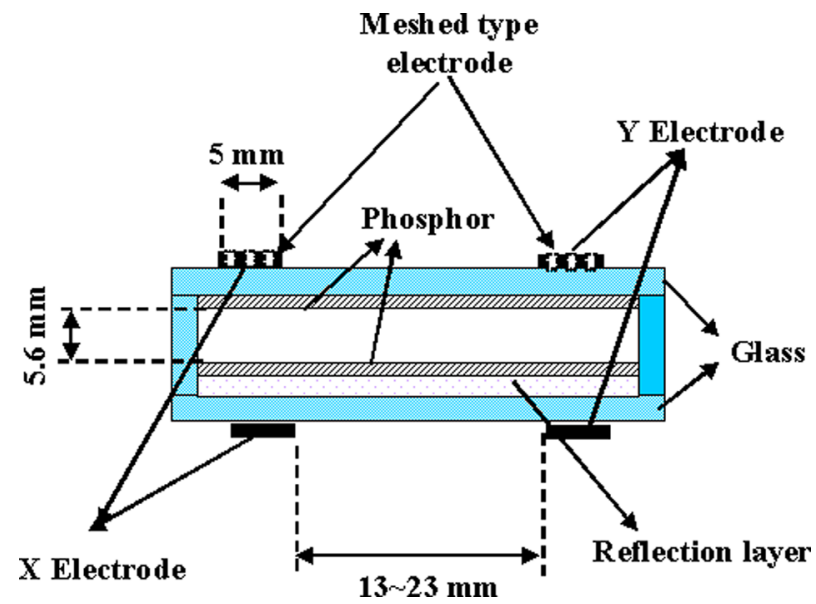

Fig. 1. Schematic diagram of the dual coplanar flat lamp. (Color version available online at http://ieeexplore.iee.org.)

addition, the results also indicate a large improvement in the brightness and luminous efficacy for this dual coplanar lamp, when compared to a conventional coplanar lamp.

\section{EXPERIMENTAL}

Fig. 1 shows a schematic diagram of the dual coplanar flat lamp. For convenience, conductive tape strips were attached as electrodes on the outer surfaces of the panel. Two electrode lines on the left side have the same electrical polarity (X), and two on the right side have the opposite polarity (Y). Xenon was used as a discharge gas with neon as a buffer gas. Various pressures and mixing ratios were tested. For the Xe discharge, the VUV radiation mainly consists of a Xe atomic resonance line at $147 \mathrm{~nm}$ and the $\mathrm{Xe}_{2}^{*}$ dimer emission at $173 \mathrm{~nm}$. For the Xe partial pressure $(>15$ torr)and lamp dimension $(>1 \mathrm{~mm}$ ) of the levels usually used in Xe back-lights and in the flat lamp in this paper, more than $90 \%$ of the radiation is the $\mathrm{Xe}_{2}^{*}$ dimer emission centered at $173 \mathrm{~nm}$ [6], [7]. The driving of the panel was performed with ac pulses of various frequencies, duty ratio, and peak voltage values. By changing the position of the conductive tape strips, we varied the inter-electrode distance from 13 to $23 \mathrm{~mm}$. The height of the sealing frame, width and length of the electrodes were fixed as $5.6,5$, and $50 \mathrm{~mm}$, respectively. The electrodes in the front plate are made in the form of meshed type to minimize the lost of light. The front and bottom plates are made of PD-200 glass of a thickness $2.1 \mathrm{~mm}$ and a dielectric constant 7.9. The white phosphor was made by mixing red ((Y,Gd)BO3:Eu), green $(\mathrm{Zn} 2 \mathrm{SiO} 4: \mathrm{Mn})$, and blue $\left(\mathrm{BaMgAl}_{14} \mathrm{O}_{23}: \mathrm{Eu}\right)$ phosphors with a mixing ratio $1: 1: 0.6$, which was determined to make the color coordinate of the final visible light from the panel to be 


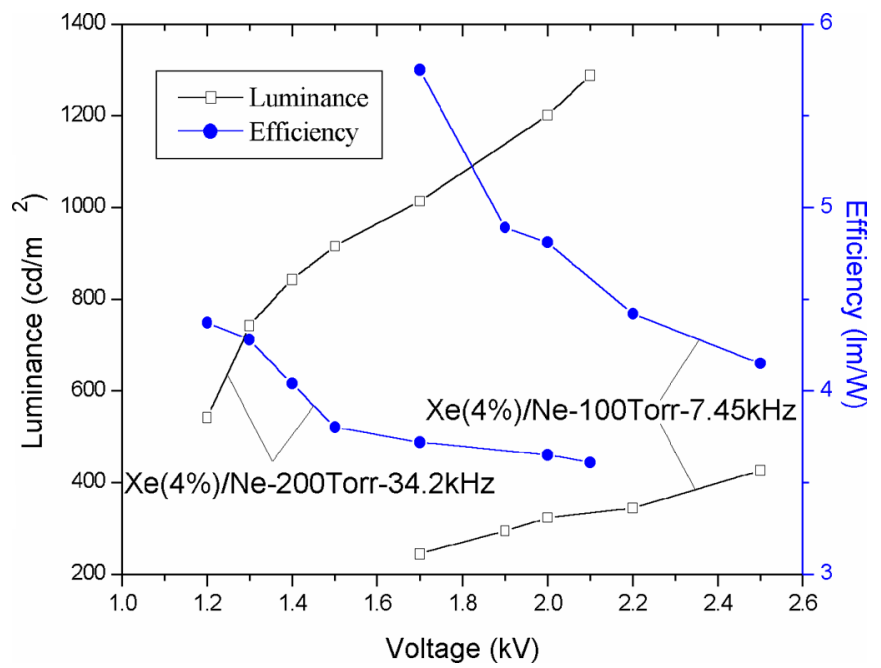

Fig. 2. Luminance and luminous efficacy in operating voltage ranges for two discharge conditions of a dual coplanar lamp: the inter-electrode distance is $18 \mathrm{~mm}$; the luminance and luminous efficacy are presented for the whole operating ranges in each case. (Color version available online at http://ieeexplore.ieee.org.)

$(0.31,0.33)$. The thickness of the phosphor layer was $0.01 \mathrm{~mm}$ for the front plate and $0.1 \mathrm{~mm}$ for the bottom plate. A $0.02-\mathrm{mm}$ reflective layer of white dielectric material was formed between the bottom phosphor and the bottom plate. For comparison, we also measured the performance of conventional coplanar electrode lamps, whose structure was the same as the lamp in Fig. 1, but with the electrodes only on the outside surface of the bottom plate.

\section{RESULTS AND DISCUSSION}

The operation voltage range of a lamp varies according to lamp parameters. In Fig. 2, we present the luminance and luminous efficacy in operating voltage ranges for two discharge conditions of a dual coplanar lamp. The two cases have different pressures and frequencies, while the inter-electrode distance is $18 \mathrm{~mm}$ for both cases. It is well known that the operation voltage increases with the increase of pressure. However, the operation voltage range of $\mathrm{Xe}(4 \%) / \mathrm{Ne}$ at 200 torr was relatively low compared to that of $\mathrm{Ne}(4 \%) / \mathrm{Ne}$ at 100 torr in Fig. 2 because the operation frequency of the case of 200 torr was higher than that of 100 torr. Therefore, it was found that the operation frequency plays an important role in reducing operation voltage. As can be seen in the Fig. 2, for higher frequencies, the discharge can be driven from a lower voltage, and the voltage at which the discharge becomes unstable and contracts is also lower for a higher frequency. The operating voltage window, therefore, is located in lower voltage range for higher frequency. For coplanar lamps, the lowest sustaining voltage is 100 or more volts higher, and the highest sustaining voltage is 100 or more volts lower than those of the dual coplanar lamps with the same conditions, resulting in narrower operating windows in general. Since the operating windows sometimes do not overlap for different discharge conditions and different electrode structures, we can not drive all the cases at the same voltage, and thus we always first define the operating voltage window for each case and measure the lu-

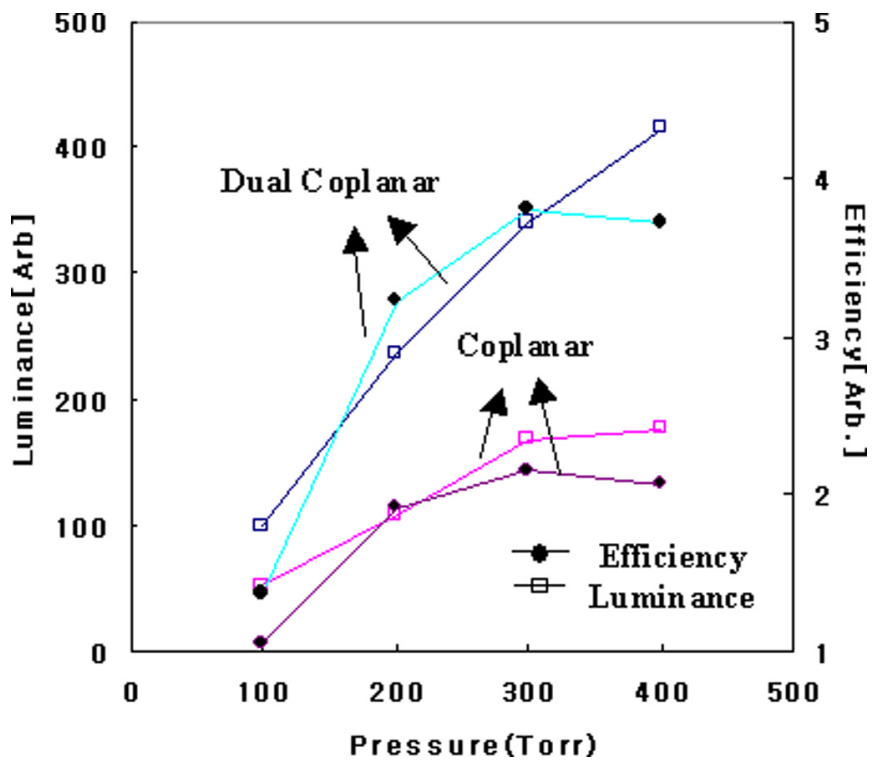

Fig. 3. Comparison of coplanar and dual coplanar lamps: $\mathrm{Xe}(4 \%) / \mathrm{Ne}, 15.2$ $\mathrm{kHz}$ ac pulses, with a peak voltage of $2.8 \mathrm{kV}$, an inter-electrode length of 18 $\mathrm{mm}$, and duty adjusted case by case so that stable discharge is established. (Color version available online at http://ieeexplore.ieee.org.)

minance and luminous efficacy at the mid-point of the operating voltage window.

Presented in Fig. 3 are experimental results of the brightness and luminous efficacy measurements for various total pressures of the $\mathrm{Xe}(4 \%) / \mathrm{Ne}$ gas mixture. For these measurements, the driving frequency of the ac pulses was $15.2 \mathrm{kHz}$, the peak voltage was set to $2.8 \mathrm{kV}$, and the pulse width was adjusted on a case by case basis so that a stable discharge was established. The inter-electrode distance was $18 \mathrm{~mm}$, and the conductive tape strips at both sides were at least $5 \mathrm{~mm}$ apart from the nearest sidewalls to avoid any extra diffusion loss of charged particles to the walls. Both the brightness and efficacy are higher for the dual coplanar lamp than for the coplanar lamp. Moreover, the breakdown voltage was also lower for the dual coplanar lamp. In our example, for the conditions described above, the dual coplanar lamp could be driven with a peak voltage of $1.2 \mathrm{kV}$ when the frequency was $34.2 \mathrm{kHz}$, while the coplanar lamp requires more than $1.3 \mathrm{kV}$. One possible reason for the lower breakdown voltage for the dual coplanar lamp is that the electric field lines are bent more in a coplanar lamp than they are in dual coplanar lamps. From a calculation of potential distribution, the result of which is given in Fig. 4, we can see that even a field line just above a dielectric surface is also varied by the electrodes on the opposite plate. The electric field line of the dual coplanar lamp around the upper and lower electrode is bent less compared to that of the coplanar lamp. Electron multiplication in the breakdown stage

$$
\Gamma_{e}(z)=\Gamma_{e}(0) \exp (\alpha z)
$$

occurs along the field lines and the effective "d" in Paschen's pd law

$$
V_{b}=\frac{B p d}{\ln A p d-\ln \left[\ln \left(1+\frac{1}{\gamma_{s e}}\right)\right]}
$$




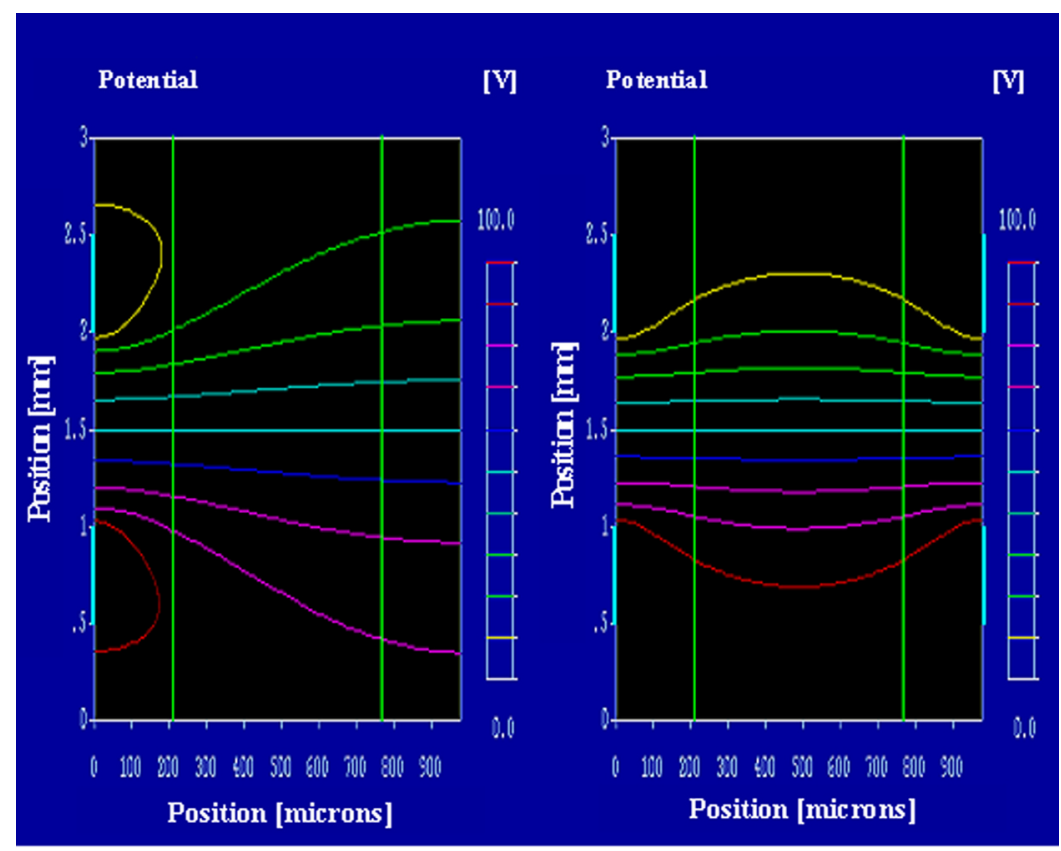

Coplanar

Dual Coplanar

Fig. 4. Vacuum electric potential distribution: the electric field lines are bent more for the coplanar lamp than for the dual coplanar lamp. (Color version available online at http://ieeexplore.ieee.org.)

for the coplanar lamp is longer than that for the dual coplanar lamp, resulting in larger pd value and higher breakdown voltage, even though the inter-electrode distance is the same for both lamps. $\Gamma_{\mathrm{e}}(\mathrm{z})$ is an electron flux at $z$ position between the cathode and anode. Here, $V_{\mathrm{b}}$ stands for breakdown voltage, $\alpha$ is a first electron emission coefficient depending on discharge gas, $p$ is the gas pressure, $d$ is the distance between the $\mathrm{X}$ and $\mathrm{Y}$ electrodes, $A$ and $B$ are constant, and $\gamma_{\mathrm{se}}$ is a second electron emission coefficient depending on cathode material. In the actual situation, the breakdown does not occur along the "single" shortest field line, although, if we compare two cases of bent and straight field line bundles, the average electron paths are longer in the former case, and the above discussion makes sense. The other possible reason for the lower breakdown voltage for the dual coplanar lamps is that the number of priming particles in the discharge volume at the early stage of the breakdown period can be larger for dual coplanar lamps than for conventional coplanar lamps. This can happen mainly because of the larger electrode area of dual coplanar lamps. The number of priming particles is also regarded to be important in the lower breakdown voltage for higher frequencies. For higher frequencies, as voltage pulses are continuously applied to the discharge volume, more priming particles produced in the previous pulse periods survive to the next period and this accumulation can induce lower breakdown voltage, as in Fig. 2.

Figs. 5 and 6 show the dependence of the brightness and luminous efficacy on the total pressure, for the dual coplanar lamp. We used a $\mathrm{Xe}(4 \%) / \mathrm{Ne}$ gas mixture and the driving frequency was $15.2 \mathrm{kHz}$. Both the peak voltage of the ac pulses as well as the pulse width was adjusted on a case by case basis so that a stable discharge was established. The inter-electrode distance $d$ varied from 13 to $23 \mathrm{~mm}$. For $d=18 \mathrm{~mm}$, the brightness and luminous efficacy were measured for two cases. In the first

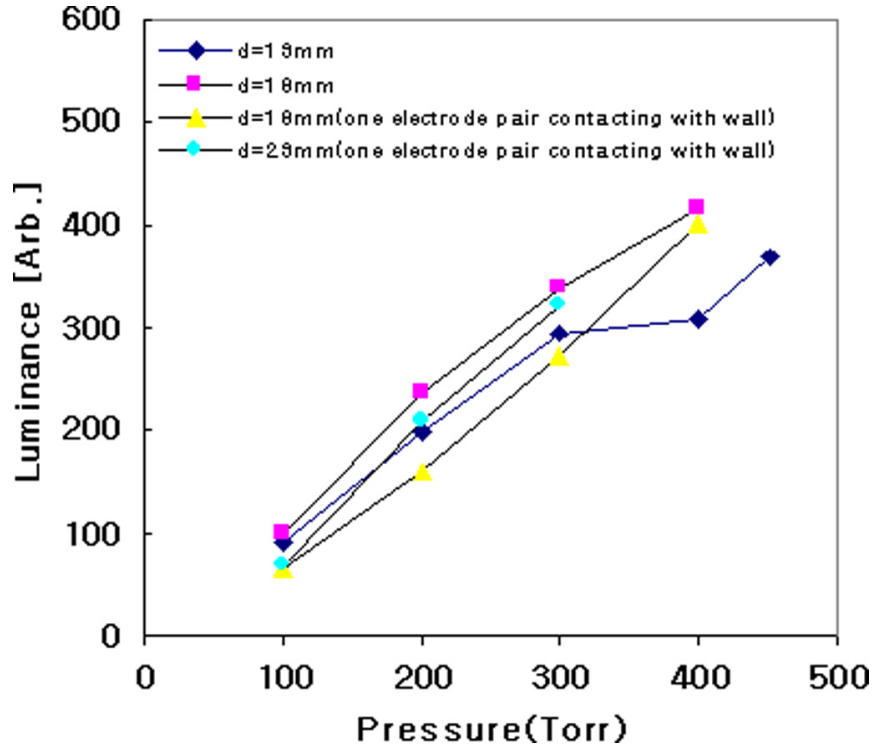

Fig. 5. Luminance of a dual coplanar lamp: $\mathrm{Xe}(4 \%) / \mathrm{Ne}, 15.2 \mathrm{kHz}$ ac pulses, peak voltage, and pulse width adjusted case by case so that stable discharge is established. (Color version available online at http://ieeexplore.ieee.org.)

case, the conductive tape strips at both sides were at least $5 \mathrm{~mm}$ apart from the nearest side walls. In the other case, we arranged the strip at one side so it would make contact with a side wall, to see the effect of diffusion loss of charged particles to the side wall. As is usual, both brightness and luminous efficacy increased with the increasing pressure. Comparing the two cases with $d=18 \mathrm{~mm}$, we noted that the diffusion loss at the side wall is not negligible. It indicates that we need a special treatment for electrodes nearest to walls. For example, when we arrange dual coplanar electrodes in a series for a large-sized lamp, we could put the electrodes on at both ends a certain distance apart 


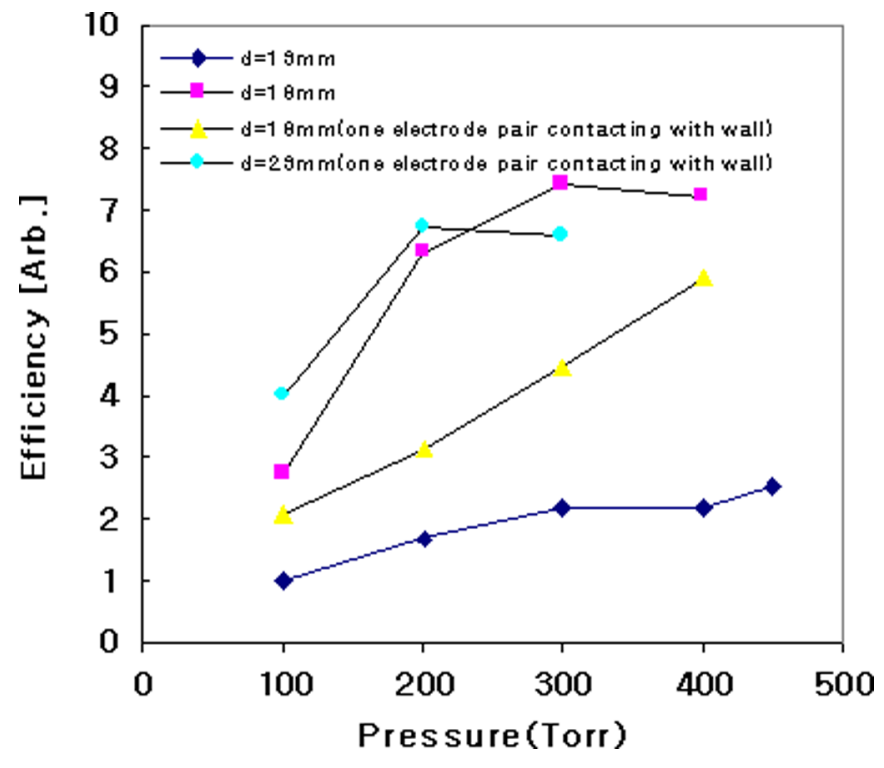

Fig. 6. Luminous efficacy of a dual coplanar lamp: the same conditions as those in Fig. 5. (Color version available online at http://ieeexplore.iee.org.)

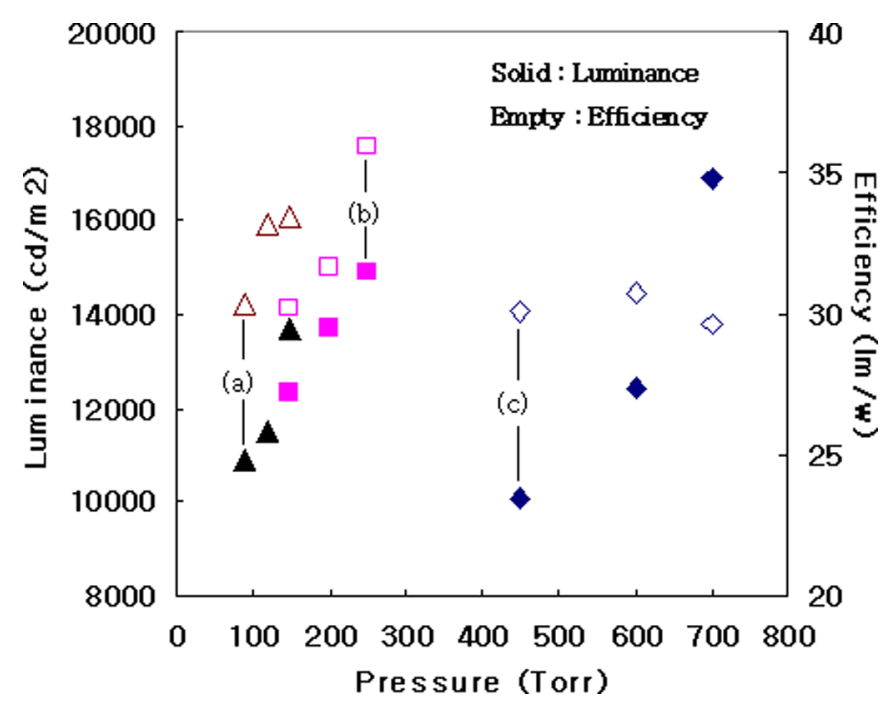

Fig. 7. Luminance and luminous efficacy of the dual coplanar lamp: (a) $\mathrm{Xe}(10 \%) / \mathrm{Ne}$, (b) $\mathrm{Xe}(30 \%) / \mathrm{Ne}$, (c) $\mathrm{Xe}(50 \%) / \mathrm{Ne}, 20 \mathrm{kHz}$ AC pulses, peak voltage $2.8 \mathrm{kV}$. (Color version available online at http://ieeexplore.ieee.org.)

from the walls. The distance can be determined so that it is long enough not to induce a large diffusion loss, but short enough not to result in dark region near the walls. Using hollow electrodes as the end electrodes [8] is another possible solution to this problem.

Fig. 7 shows the luminance and luminous efficacy of the dual coplanar lamp for $\mathrm{Xe}(10 \sim 50 \%) / \mathrm{Ne}$ gas mixtures as a function of the total gas pressure. The highest efficacy of $35.9 \mathrm{~lm} / \mathrm{w}$ $\left(14900 \mathrm{~cd} / \mathrm{m}^{2}\right)$ was obtained for 250 torr of the $\mathrm{Xe}(30 \%) / \mathrm{Ne}$ gas mixture.

Fig. 8 shows the photograph of the dual coplanar lamp with discharges. The driving conditions of the lamp in Fig. 8 are the same as those of Fig. 7(c).

In Figs. 9 and 10, we present the results from the numerical simulations for conventional coplanar lamps and dual coplanar lamps. The simulations were done for the structure of Fig. 1,

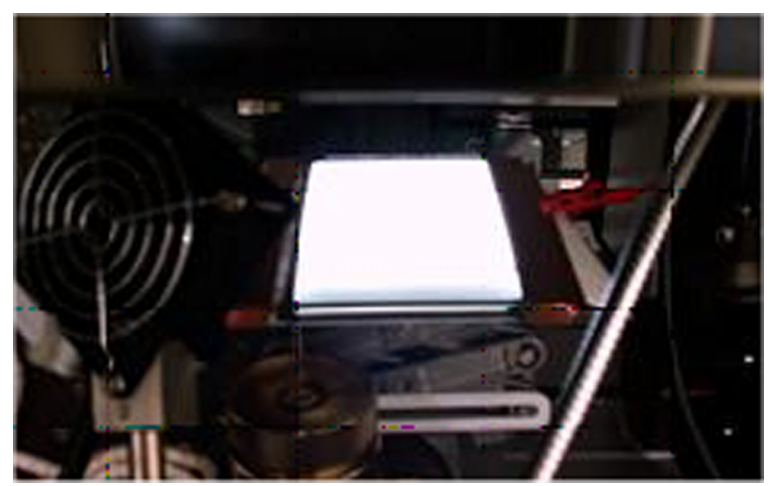

Fig. 8. Photograph of the 6 inch-dual coplanar lamp with discharges. (Color version available online at http://ieeexplore.ieee.org.)

with only the bottom electrodes for the coplanar lamps and both the front and bottom electrodes for the dual coplanar lamps. They were performed with a two-dimensional fluid code SIPDP-AC [9]. The size of the panels was reduced in the simulations to avoid a failure of the code in resolving the sheath (the nonneutral potential region between the plasma and the wall [10]) in case the $p d$ product is too large. This could occur in the case where the length of sheath shrinks and becomes too small to be calculated as the gas pressure (p) and distance (d) increase. An inter-electrode distance of $1 \mathrm{~mm}$, an electrode width of $0.5 \mathrm{~mm}$, a distance from electrode to the nearest side wall of $0.5 \mathrm{~mm}$, and a height (of the panel) of $0.5 \mathrm{~mm}$ were used. The two-dimensional code assumes an infinite homogeneity along the direction of the electrodes, thus the length of the electrodes was not needed in the simulation. The total pressure was also reduced to 100 torr. The boundary conditions on the sides of the simulation domain perpendicular to the electrodes were that the electric field and the charged particle fluxes perpendicular to these boundaries were set to zero. On the sides of the simulation domain parallel to the electrodes (corresponding to the interface between the cell and the glass plate), the potential was set to the electrode voltages where the electrodes were present. Between the electrodes on the same side the perpendicular electric field was set to zero. This imposed the condition that no (displacement) current flowed out of the simulated domain. The total charge $\sigma$ per unit surface of the dielectric walls was obtained by assuming that electrons and ions recombine instantaneously on a perfectly absorbing boundary. At the interface between the gas and any dielectric surface the charge density was calculated by integrating the charged particle current to the surface during the evolution of each discharge pulse. At $t=0$, the potential distribution was determined by solving Poisson's equation in the absence of space charge. This was just an initial condition. During the calculation, the space charge effect was considered, as plasmas were generated. The final boundary condition needed to fully define the problem was the relation between the electron current leaving the cathode and the incident ion current. These quantities are related through the secondary electron emission coefficient, $\gamma$. Because of the difficulty in measuring the secondary electron emission coefficients and their dependences on various discharge conditions, the exact secondary electron emission coefficients for $\mathrm{Xe}$ and $\mathrm{Ne}$ ions were not known at 


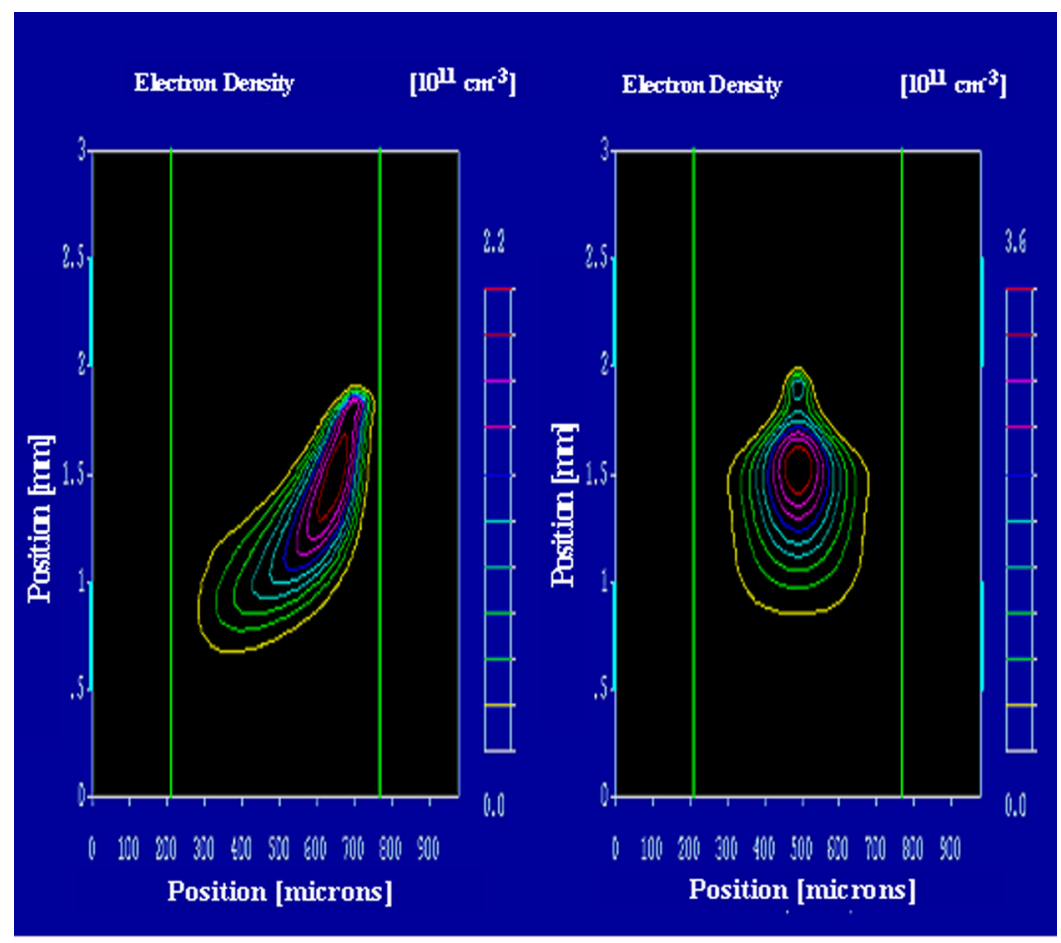

\section{Coplanar}

\section{Dual Coplanar}

Fig. 9. Electron distribution: inter-electrode distance of $1 \mathrm{~mm}$; electrode width of $0.5 \mathrm{~mm}$; distance from electrode to nearest sidewall of $0.5 \mathrm{~mm}$; cell gap of 0.5 $\mathrm{mm}$; total pressure of 100 torr; secondary electron emission coefficients for Xe and $\mathrm{Ne} 0.01$ and 0.3 , respectively; rectangular ac driving pulses with peak voltage of $1 \mathrm{kV}$; period of $12 \mu \mathrm{s}$; and pulse width of $2 \mu \mathrm{s}$. (Color version available online at http://ieeexplore.ieee.org.)

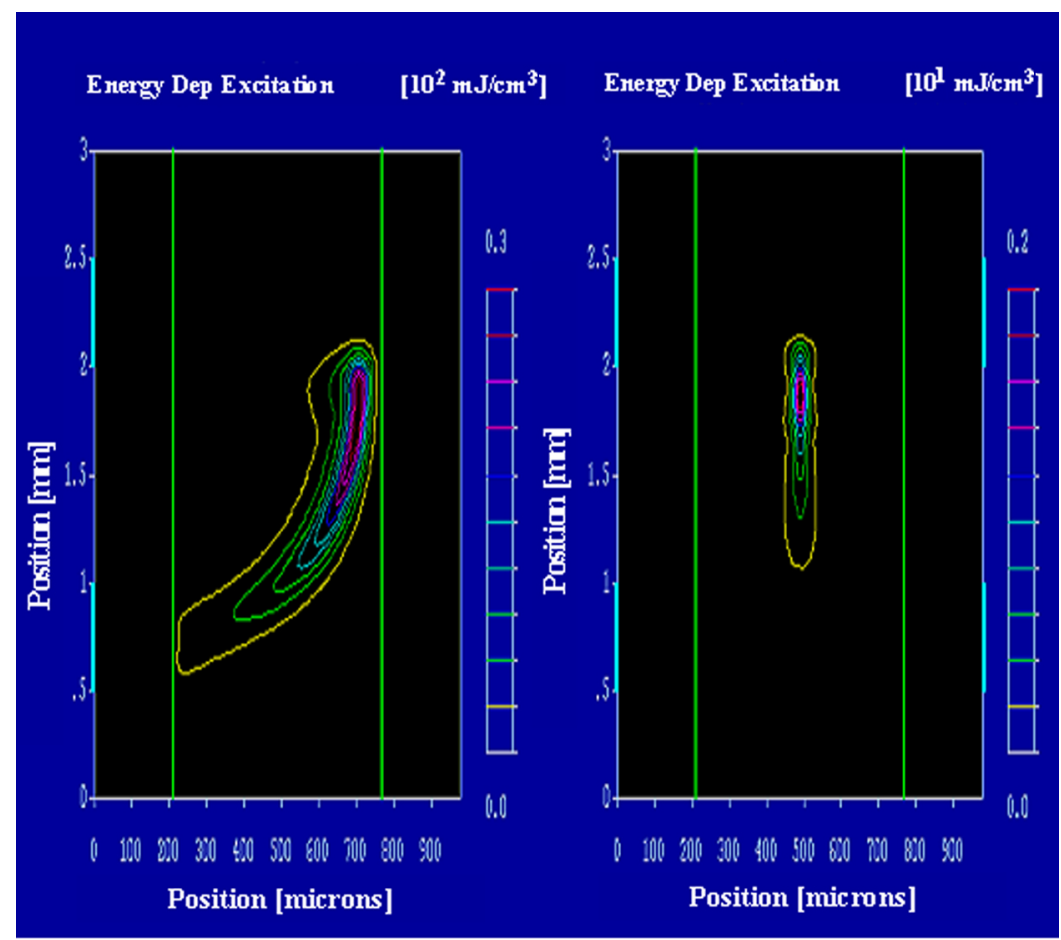

Coplanar

Dual Coplanar

Fig. 10. Energy spent on Xe excitation: same conditions as those in Fig. 9. (Color version available online at http://ieeexplore.ieee.org.)

this point and were assumed as different values in different simulation tools [9], [11]. For SIPDP-AC[9], [12], used for simulation in this paper, the secondary electron emission coef- ficients were usually set to 0.05 and 0.5 for the Xe and Ne ions on MgOsurfaces, respectively. Here, we assumed the secondary electron emission coefficients for $\mathrm{Xe}$ and $\mathrm{Ne}$ ions as 0.01 and 


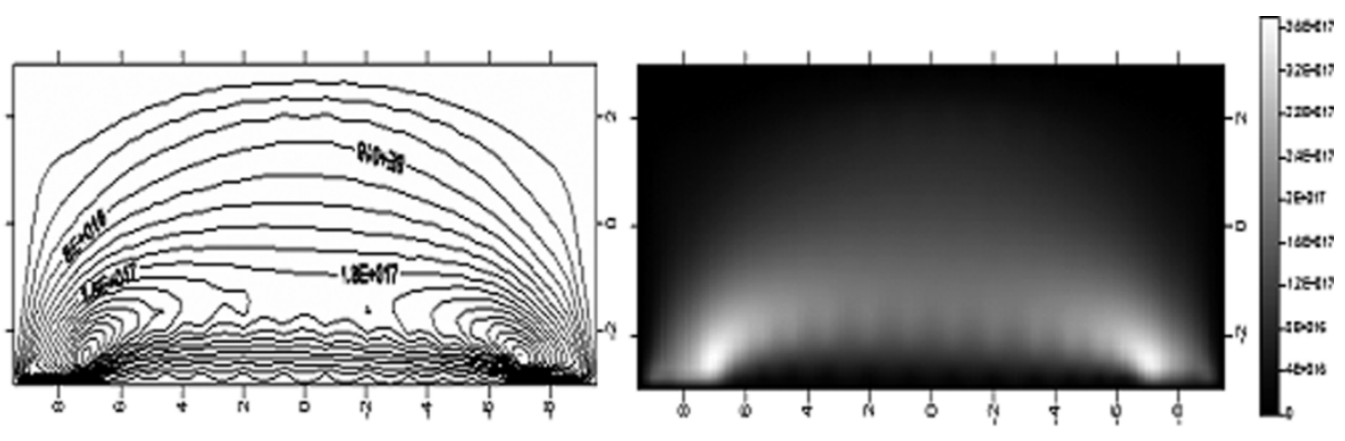

Coplanar
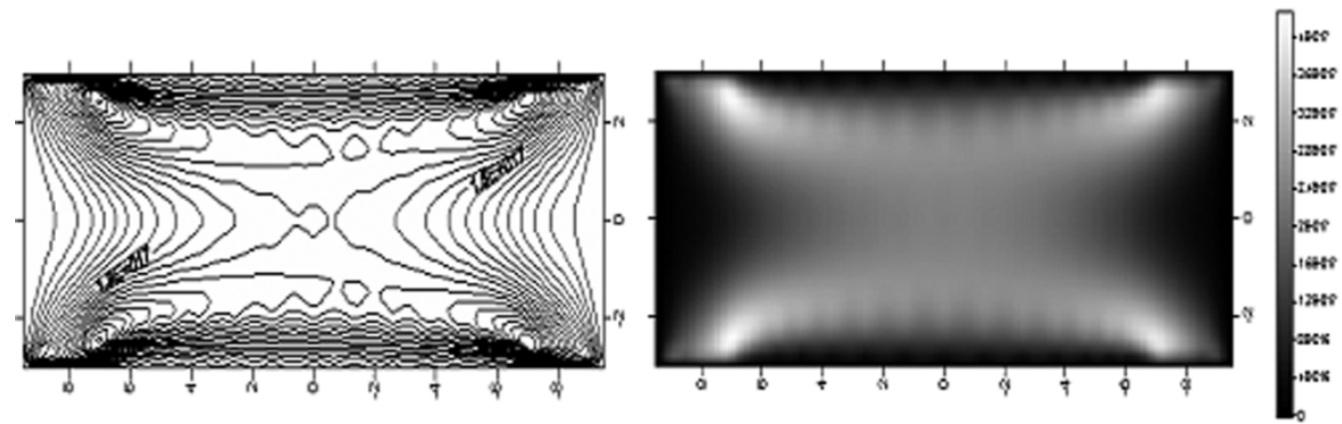

\section{Dual Coplanar}

Fig. 11. Time-averaged electron density distribution over a half pulse period for coplanar and dual coplanar lamp. Simulation conditions are the same as in Fig. 9, but with a higher Xe partial pressure (pure Xe 100 torr), and higher cell gap of $6 \mathrm{~mm}$.
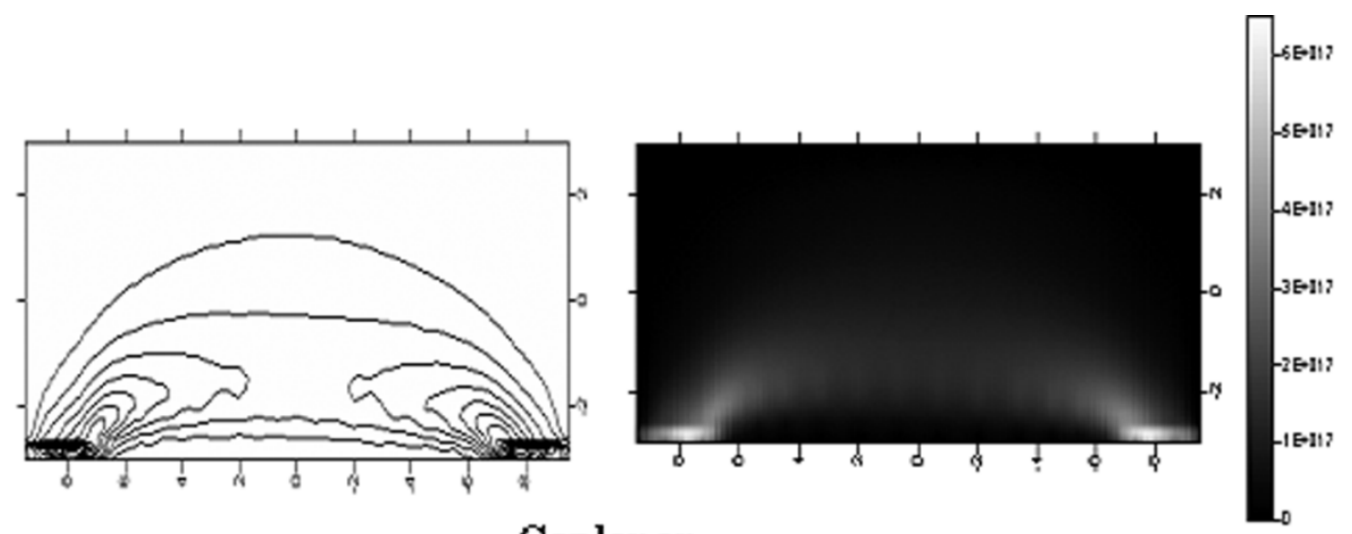

\section{Coplanar}
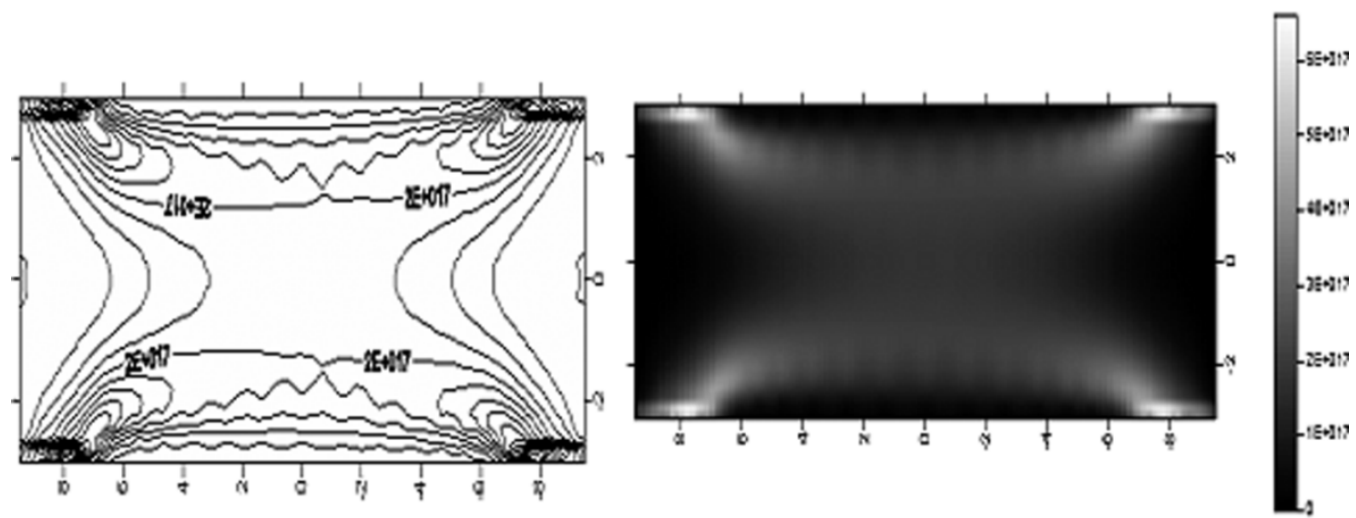

\section{Dual Coplanar}

Fig. 12. Time-averaged $\mathrm{Xe} *\left({ }^{3} \mathrm{P}_{1}\right)$ density distribution over a half pulse period for the coplanar and the dual coplanar lamps. Simulation conditions are the same as in Fig. 11.

0.3 , respectively, on phosphor surfaces. Qualitative behaviors such as the shape of density distributions, relative superiority of the dual coplanar structure to coplanar structure were not changed much by varying the coefficients within reasonable 
ranges. Rectangular ac driving pulses with a peak voltage of $1 \mathrm{kV}$, a period of $12 \mu \mathrm{s}$ and a pulse width of $2 \mu$ s were used in the simulations. Fig. 9 shows the electron distributions for the coplanar and dual coplanar lamps. Each figure for coplanar and dual coplanar lamps presents the electron distribution at the moment when the electron energy deposition efficiency into the Xe excitation accumulated from the beginning of the voltage pulse is highest. This moment differs between cases and generally occurs during a falling period of current pulses. The distribution for the dual coplanar lamp is more centered than that of the coplanar lamp. From this, it can be expected that the diffusion loss of charged particles will be smaller for dual coplanar lamps than for coplanar lamps. This is what we expected from the dual coplanar structure for better results in brightness and efficacy, in this low pressure and/or low cell gap range.

Fig. 10 shows the distributions of energy spent in Xe excitation by electrons for the coplanar and dual coplanar lamps. The energy in this figure is an accumulated value from the beginning of the voltage pulse to the moment when the accumulated electron energy deposition efficiency into the Xe excitation is the highest. Since the cell structure in the simulations was the same for both kind of lamps, comparing the energy spent in Xe excitation is virtually the same as comparing the brightness of the lamps. The value or the excitation energy is larger and the distribution is more centered, as can be expected from the electron distributions for the dual coplanar lamp.

For higher pressure and/or a larger dimension, usual discharge display simulators cannot deal with the problem and a grid system in which the grid size varies in discharge volume is inevitable. In Figs. 11 and 12, we show results of the macro-discharge simulation for the above cases shown in Figs. 9 and 10, but with a higher Xe partial pressure (pure Xe 100 torr) and a higher cell gap of $6 \mathrm{~mm}$. Fig. 11 is the time-averaged electron density distribution over a half pulse period and Fig. 12 is the time-averaged $\mathrm{Xe}^{*}\left({ }^{3} \mathrm{P}_{1}\right)$ density distribution over a half pulse period. These were obtained using the simulation tool for macro-discharge light source based on the CFD-ACE package. In this high-pressure range, the electron and excited species distributions are bent less, and the diffusion loss can be reduced even for conventional coplanar lamps compared to the low pressure case if the cell gap is high enough. However, a coplanar lamp can not use the discharge volume sufficiently because $\mathrm{Xe}^{*}\left({ }^{3} \mathrm{P}_{1}\right)$ distribution at phosphor area of upper plate is less dense compared that of the dual coplanar lamp, as shown in Fig. 12. This is true in the case of electron density as shown in Fig. 11. From these results, it was found that the dual coplanar lamp utilized the VUV emitted from $\mathrm{Xe}^{*}\left({ }^{3} \mathrm{P}_{1}\right)$ more effectively compared to a coplanar lamp.

In general, a plane parallel electrode discharge produces a higher luminance and luminous efficacy than a coplanar electrode discharge, because of less diffusion loss of charged particles compared to the coplanar discharge. On the other hand, the stability of the plane parallel discharge is often less than that of the coplanar discharge because of the higher current, especially in an $\mathrm{Hg}$-free lamp that may use a rare gas mixture. The purpose of the dual coplanar lamp is to combine the good points of both coplanar and plane parallel electrode discharges.
Near the electrodes, the electric field structure is the same as that in a coplanar electrode discharge, meaning the discharge is stable and there exists less sputtering compared to plane parallel discharges. On the other hand, in the bulk region the field is straight parallel to the front and bottom plates, similar to that in a plane parallel discharge, which results in less diffusion loss compared to a coplanar discharge, raising the brightness and luminous efficacy.

\section{CONCLUSIONS}

The authors introduced a dual coplanar mercury-free flat fluorescent lamp and presented experimental results showing that the brightness and efficiency are almost doubled for the dual coplanar lamp compared to a conventional coplanar lamp. The efficacy of $35.9 \mathrm{~lm} / \mathrm{w}\left(14900 \mathrm{~cd} / \mathrm{m}^{2}\right)$ was achieved for 250 torr of $\mathrm{Xe}(30 \%) / \mathrm{Ne}(70 \%)$ gas mixture, by a $20-\mathrm{kHz}$ ac pulse driving. Numerical analyzes indicated that the improvements in brightness and efficiency resulted mainly from the less bent electric field lines for the dual coplanar lamp. This resulted in fewer electrons near the boundary, and less diffusion loss for the dual coplanar lamp in a low pressure and/or a low cell gap range. As well it resulted in a more effective usage of discharge volume in the dual coplanar lamps, for which the volume used for discharge was larger and the distance between the produced VUV and phosphor is shorter in a high pressure and/or a high cell gap range.

\section{REFERENCES}

[1] Y. Ikeda, T. Shiga, and S. Mikoshiba, "Mercury-free, simple-structured flat discharge LCD backlights ranging from 0.5 to 5.2-in. diagonals," in SID Int. Symp. Dig. Tech. Papers, 2000, pp. 938-941.

[2] M. Ilmer, R. Lecheler, H. Schweizer, and M. Seibold, "Hg-free flat panel light source PLANON, a promising candidate for future LCD back lights," in SID Int. Sym. Dig. Tech. Papers, 2000, pp. 931-934.

[3] T. Sakurai, T. Saikatsu, and T. Myodo, "VUV radiation of the rare gas discharge fluorescent lamp at pulsed discharge," in Proc. 6th Int. Symp. on the Science \& Technology of Light Sources, 1992, pp. 119-120.

[4] S. Mikoshiba, "Xe-discharge backlights for LCDs," in SID Int. Sym. Dig. Tech. Papers, 2001, pp. 286-289.

[5] M. G. Kwak, J. L. Han, W. K. Kim, S. K. Park, S. J. Hong, Y. H. Kim, J. Y. Choi, K. S. Jeong, and S. H. Sohn, "Mercury-free $18^{\text {" class flat }}$ fluorescent lamp with good uniformity," in SID Int. Symp. Dig. Tech. Papers, 2001, pp. 391-393.

[6] R. T. Mcgrath, R. Veerasingam, J. A. Hunter, P. D. Rockett, and R. B. Campbell, "Measurements and simulations of VUV emission from plasma flat panel display pixel microdischarges," IEEE Trans. Plasma Sci., vol. 26, no. 5, pp. 1532-1541, Oct. 1998.

[7] T. Holstein, "Imprisonment of resonance radiation in gases, II," Phys. Rev., vol. 83, no. 6, pp. 1159-1167, 1951.

[8] M. Anandan, D. Ketchum, H. Etlinger, R. Kirker, and W. Carr, "Design of a multi-channel true flat fluorescent lamp for avionic AMLCD back lighting," IEEE Trans. Electron Devices, vol. 41, no. 4, pp. 504-511, Apr. 1994.

[9] C. Punset, S. Cany, and J. P. Boeuf, "Addressing and sustaining in alternating current coplanar plasma display panels," J. Appl. Phys., vol. 86, pp. 124-133, 1999.

[10] M. A. Liberman and A. J. Lichtenberg, Principles if Plasma Discharges and Material Processing. Hoboken, NJ: Wiley, 2005.

[11] W. J. Chung et al., "Mechanism of high luminous efficient discharges with high pressure and high Xe-content in AC PDP," IEEE Trans. Plasma Sci., vol. 31, no. 5, pp. 1038-1043, Oct. 2003.

[12] J. Ouyang, T. Callegari, N. Lebarq, B. Caillier, and J.-P. Boeuf, "Plasma display panel cell optimization: modeling and macro-cell experiments," in Proc. Eur. Display 2002, 2002, pp. 53-56. 


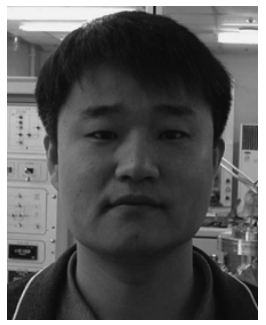

Hyoung-Bin Park received the B.S., M.S., and Ph.D. degrees in 1991, 1993, and 1998, respectively, from Korea Advanced Institute of Science and Technology (KAIST), Suwon, Korea.

During his Ph.D. degree, he worked on the transport simulation studies of plasmas. After his graduation, he joined National Institute for Fusion Science in Japan and worked as a visiting researcher on Fokker-Planck simulation of fusion plasmas. Following his work on high temperature fusion plasma, he joined Samsung Advanced Institute of Technology in Korea as a senior researcher for the discharge display research group and developed high efficiency discharge display devices, including plasma display and mercury free flat discharge backlight for LCD. He recently joined Samsung SDI as a senior researcher. His main areas of interests are the improvement of plasma display in luminous efficiency and picture quality, and the development of a new concept next generation information display. $\mathrm{He}$ has published more than 40 papers in journal and conference proceedings and applied more than 60 patents.

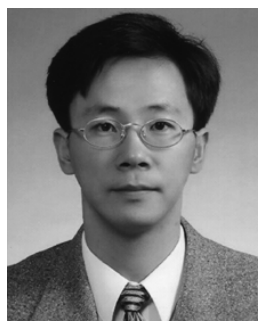

Seong-Eui Lee received the B. S., M.S., and Ph.D. degrees in 1988, 1990, and 1995 , respectively, from Seoul National University, Seoul, Korea.

During his Ph.D degree, he worked on the phase transformation kinetics of amorphous powder. After his gradation, he joined Hyundai Electronics Korea and worked as a senior researcher at Hyundai Plasma Display research center in Japan for 26-in XGA PDP project. After his PDP work, he joined iFire Technology Inc. as a senior researcher for the thick film technology development group. After returning to Korea, he worked as a principal engineer at Samsung Advanced Institute of Technology for thick-film related display development especially PDP \& flexible display. He recently joined Korea Polytechnic University as a faculty member at the department of Advanced Materials Engineering. His main areas of interests are the process development and integration for display based on thick film or printing technology. He has published more than 20 papers in journal and conference proceedings and hold more than 40 patents.

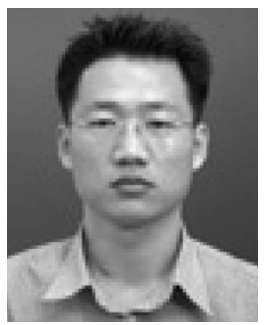

Gi Young Kim received the B.S. and M.S. degrees from Kyunghee University, in 1997 and 1999, respectively.

During his M.S. degree, he worked on the Optical fiber Raman Laser used by Nd:YAG Laser. After his graduation, he joined Samsung Advanced Institute of Technology and worked as a researcher at Display Laboratory of Materials and Devices Sector in 1999. $\mathrm{He}$ worked on the high luminous efficacy of PDP during the next five years. He also joined $\mathrm{Hg}$-free LCD backlight Project team from 2000, and recently joined R\&D 2 Team Corporate R\&D Center of Samsung SDI. His main areas of interests are the process development \& integration for display based on thick film or printing technology. He has published more than 20 papers in journal and conference proceedings and hold more than 60 patents.

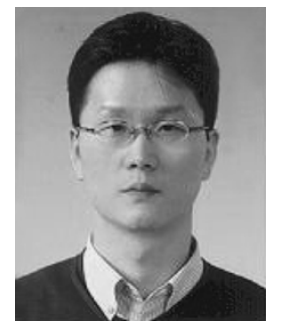

Young Dong Lee was born in May 17, 1970. He received the B.S. degree from Pusan National University, Pusan, South Korea, and the M.S. and the Ph.D. degrees in plasma physics from the Korea Advanced Institute of Science and Technology, Taejon, South Korea, in 1994 and 2000, respectively. $\mathrm{He}$ is currently with Manufacturing Equipment R\&D Team, Samsung Electronics, Suwon, Korea, as a senior researcher. His research interest is in the area of plasma application technology.

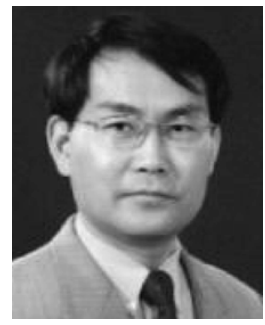

Kyung Cheol Choi (M'04) received the B.S. degree in electrical engineering, in 1986, and the M.S. and $\mathrm{Ph} . \mathrm{D}$. degrees in plasma engineering from in 1988 and 1993, respectively, all Seoul National University, Seoul, Korea

He was with the Institute for Advanced Engineering, Seoul, from 1993 to 1995 , where his work focused on the design of field emission display devices. He was a Research Scientist in the Microbridge Plasma Display panel of Spectron Corporation of America, Summit, NJ, from 1995 to 1996. He was a Senior Research Scientist at Hyundai Plasma Display, Hawthorne, NY, from 1996 to 1998, where his work was to continue on developing plasma display technology. From 1998 to 1999, he was involved in the development of an ac 40-in Plasma Display Panel at Advanced Display R\&D center of Hyundai Electronics Industries, Gyounggi-do, South Korea, as a Senior Research Scientist. From 2000 to 2004, he had been an associate professor in the Department of Electronics Engineering, Sejong University, Seoul, Korea. He also was in charge of the Information Display Research Center supported by Korean Ministry of Information and Communication. Since February 1, 2005, he has been an associate professor in the Department of Electrical Engineering \& Computer Science, Korea Advanced Institute of Science and Technology (KAIST), Daejeon, Korea. His research interests include plasma display panel, information displays, and micro-plasma applications for laser and bio-electronics.

Dr. Choi is a member of the Society for Information Display and the Korean Information Display Society. 